\title{
A SIMPLE THEORY OF WEAKLY INHOMOGENEOUS FLUIDS
}

\author{
A.G. Schlijper and B. Smit \\ Koninklijke/Shell Exploratie en Produktie Laboratorium \\ Volmerlaan 6, 2288 GD Rijswijk ZH (The Netherlands)
}

Keywords: Inhomogeneous fluids, integral equations, cluster variation method.

\section{ABSTRACT}

A theory for the description of the thermodynamic behaviour of inhomogeneous fluids is derived by the mathematical equivalent of the cluster variation method for lattice systems. A systematic expansion of the free energy functional is generated, which is then truncated and minimised to obtain integral equations for the density profile and the pair distribution function. The theory contains no adjustable parameters, the only input is the intermolecular potential and the external field (which includes wall potentials).

A simplified version of this theory, valid for weakly inhomogeneous fluids, is investigated by applying it to hard-sphere and Lennard-Jones fluids between parallel plates and comparing the results with computer simulations.

\section{INTRODUCTION}

This paper applies an adaptation of the Cluster Variation Method (CVM) to the theory of liquids. The CVM was originally proposed by Kikuchi (Kikuchi, 1951), and its more recent and general formulations (Schlijper, 1983; An, 1988) have shown that it provides a comprehensive formalism for the generation of approximate theories of lattice models. In the CVM formalism a Möbius transformation (Rota, 1964) is used to generate a cumulant expansion for the free energy of the model, and approximate theories result from minimisation of truncated versions of this expansion.

In previous publications an adaptation of this CVM formalism to the theory of fluids was presented (Schlijper \& Kikuchi, 1990; Schlijper \& Harris, 1991). Here the formalism is used to derive a simple theory for weakly inhomogeneous fluids. The theory takes the form of an integral equation for the local density. It is derived by the following procedure. First, the free energy of the system of $\mathrm{N}$ particles in a volume $\mathrm{V}$ is written as a cumulant expansion. This expansion is truncated after 3 
terms. The resulting approximation to the free energy is a functional of the 3-body density function $\rho^{(3)}\left(\vec{r}_{1}, \vec{r}_{2}, \vec{r}_{3}\right)$.

Minimisation yields an equilibrium equation for $\rho^{(3)}$, which can be rewritten as a set of equations for the local density and the pair distribution function $\mathrm{g}^{(2)}$. Up to this point the procedure parallels the treatment of a lattice model by the CVM. The difference and major technical difficulty is that the equations involve the system size in a non-trivial way; it is necessary to extract the large $\mathrm{N}$ behaviour, while the average density $\rho=N / V$ is kept fixed. This takes a significant calculational effort that is not reproduced here but can be found in (Schlijper \& Harris, 1991).

The procedure results in a set of coupled nonlinear integral equations, which is simplified in section 3 by an additional approximation. This leads to a comparatively simple theory that is expected to be valid for weakly inhomogeneous fluids.

In section 4 applications of the theory to hard-sphere and Lennard-Jones fluids confined to the space between plane parallel hard walls are discussed.

\section{APPROXIMATE EQUILIBRIUM EQUATIONS FOR AN (INHOMOGENEOUS) FLUID IN THE CANONICAL ENSEMBLE}

Consider $\mathrm{N}$ identical particles of mass $\mathrm{m}$ in a volume $\mathrm{V}$. The particles are numbered 1 to $N$ and they interact with $\mathrm{k}$-body potentials $\Phi^{(\mathrm{k})}\left(\overrightarrow{\mathrm{r}}_{1}, \ldots, \overrightarrow{\mathrm{r}}_{\mathrm{k}}\right), \mathrm{k}=1, \ldots, \mathrm{N}$. If the particles 1 to $N$ are at positions $\vec{r}_{1}$ to $\vec{r}_{N}$ in the volume $V$, then the potential energy of the system is

$$
E^{(N)}\left(\vec{r}_{1}, \ldots, \vec{r}_{N}\right)=\sum_{i=1}^{N} \sum_{\substack{k-t u p l e s \\\left\{i_{1}, \ldots, i_{k}\right\} \subset\{1, \ldots, N)}} \Phi^{(k)}\left(\vec{r}_{i_{1}}, \ldots, \vec{r}_{i_{k}}\right)
$$

We assume that each potential function $\Phi^{(\mathrm{k})}$ is invariant under permutation of its arguments. The one-body potential $\Phi^{(1)}$ describes an external force field.

We now consider the joint probability of finding particle $i$ in a volume element $d \vec{r}_{i}$ for $i=1, \ldots, N$ and we denote the associated probability density by $\mathrm{P}^{(\mathrm{N})}\left(\overrightarrow{\mathrm{r}}_{1}, \ldots, \overrightarrow{\mathrm{r}}_{\mathrm{N}}\right)$.

The n-particle density functions $\rho_{N}^{(n)}$ are defined by

$$
\rho_{N}^{(n)}\left(\vec{r}_{1}, \ldots, \vec{r}_{n}\right)=\frac{N !}{(N-n) !} \int_{V} d \vec{r}_{n+1} . . d \vec{r}_{N} P^{(N)}\left(\vec{r}_{1}, \ldots, \vec{r}_{N}\right)
$$

From these density functions one traditionally goes on to define the n-particle distribution functions $\mathrm{g}_{\mathrm{N}}^{(\mathrm{n})}$ as 
$g_{N}^{(n)}\left(\vec{r}_{1}, \cdots, \vec{r}_{n}\right)=\frac{\rho_{N}^{(n)}\left(\vec{r}_{1}, \ldots, \vec{r}_{n}\right)}{\sum_{i=1}^{n} \rho_{N}^{(1)}\left(\vec{r}_{i}\right)}$

but we shall work with functions

$G_{N}^{(n)}\left(\vec{r}_{1}, . ., \vec{r}_{n}\right)=\rho^{-n} \rho_{N}^{(n)}\left(\vec{r}_{1}, \ldots, \vec{r}_{n}\right)$

where $\rho$ denotes the average particle density, $\rho=N / V$.

The $\mathrm{G}_{\mathrm{N}}^{(\mathrm{h})}$ are linked through the reduction relations

$\rho \int_{V} d \vec{r}_{n+1} G_{N}^{(n+1)}\left(\vec{r}_{1}, \ldots, \vec{r}_{n+1}\right)=(N-n) G_{N}^{(n)}\left(\vec{r}_{1}, . ., \vec{r}_{n}\right)$

and they are normalised according to

$\rho^{n} \int_{V} d \vec{r}_{1} . . d \vec{r}_{n} G_{N}^{(n)}\left(\vec{r}_{1}, \ldots, \vec{r}_{n}\right)=\frac{N !}{(N-n) !}$

We introduce a shorthand notation for all these k-particle functions by dropping the subscript $N$ that refers to the size of the system and by indicating the argument list and the order (i.e. number of particles involved) by a list of subscripts and their total number. Thus, $G_{124}$ will stand for $G_{N}^{(3)}\left(\vec{r}_{1}, \vec{r}_{2}, \vec{r}_{4}\right)$, etc.

The excess internal energy of the system, $U_{N}$, can now be written as

$\mathrm{U}_{\mathrm{N}}=\sum_{\mathrm{k}=1}^{\mathrm{N}} \frac{\rho^{\mathrm{k}}}{\mathrm{k} !} \int_{\mathrm{V}} \mathrm{d} \overrightarrow{\mathrm{r}}_{1 . . \mathrm{d}} \overrightarrow{\mathrm{r}}_{\mathrm{k}} \mathrm{G}_{1 . . \mathrm{k}} \Phi_{1 . . \mathrm{k}}$

This representation derives its usefulness from the fact that the contributions of the k-body potentials $\Phi^{(k)}$ are expected to decrease rapidly with increasing k. In fact, most systems are modelled with two-body potentials only. The idea of the present approach is to isolate k-body contributions to the entropy in a similar manner, so as to provide a starting point for approximations. To do so, we define functions $\gamma_{N}^{(k)}$ by

$$
\log \gamma_{1 . . n}=\sum_{k=1}^{n} \quad(-1)^{n-k} \sum_{\substack{\left\{i_{1} . . i_{k}\right\} \\ c\{1, ., n\}}}^{\sum} \log G_{i_{1} . . i_{k}}
$$


This relationship can be inverted (Rota, 1964):

$\log G_{1 . . n}=\sum_{k=1}^{n} \sum_{\substack{\left\{i_{1} \ldots i_{k}\right\} \\ c\{1, \ldots, n\}}} \log \gamma_{i_{1} . . . i_{k}}$

The entropy of the system can be expressed, after a little algebra, in the form

$S_{N}=\frac{3}{2} N_{B}-3 N k_{B} \log \Lambda-\log \rho-k_{B} \sum_{k=1}^{N} \quad \frac{\rho_{k}}{k !} \int_{V} d \vec{r}_{1} . . d \vec{r}_{k} G_{1 . . k} \log \gamma_{1 . . k}$

with $\Lambda=h / \sqrt{ }(2 \pi \mathrm{mkT})$ the thermal wavelength. By combining Eqs. (7) and (10) we

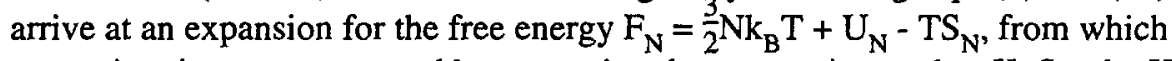
approximations are generated by truncating the summations at $\mathrm{k}=\mathrm{K}$. So, the $\mathrm{K}$-th order approximation neglects all interactions and correlations involving groups of more than $\mathrm{K}$ particles and is completely determined by the free energy functional $\mathrm{F}_{\mathrm{N}}^{(\mathrm{K})}$ :

$\beta F_{N}^{(K)}=3 N \log \Lambda+N \log \rho+\sum_{k=1}^{K} \frac{\rho^{k}}{k !} \int_{V} d \vec{r}_{1} . . d \vec{r}_{k} G_{1 . . k} \log \Psi_{1 . . k}$

where the functions $\psi_{\mathrm{N}}^{(\mathrm{k})}\left(\overrightarrow{\mathrm{r}}_{1}, \ldots, \overrightarrow{\mathrm{r}}_{\mathrm{k}}\right)$ are defined for notational convenience by

$\psi_{1 . . \mathrm{k}}=\gamma_{1 . . \mathrm{k}} \cdot \exp \left(\beta \Phi_{1 . . \mathrm{k}}\right)$

Note that $F_{N}^{(K)}$ is a functional of $G_{N}^{(K)}$ only, since this function determines all the lower-order functions $G_{N}^{(k)}$ for $\mathrm{k}=1, \ldots, \mathrm{K}-1$, through the reduction relations (5), and since the collection $G_{N}^{(k)^{N}}, k=1, \ldots, K$ determines the $\gamma_{N}^{(k)}$ (and thus the $\psi_{N}^{(k)}$ ) for $k=1, \ldots, K$, through the defining equation (8). At its minimum the free energy functional $F_{N}^{(K)}$ is stationary w.r.t. variations in $G_{N}^{(K)}$. The stationarity condition is found to be, after some straightforward algebra,

$\sum_{k=1}^{K} \frac{(K-k) !}{(N-k) !} \sum_{\substack{\left\{i_{1} \ldots i_{k}\right\} \\ c\{1, \ldots, K\}}} \log \Psi_{i_{1}, \ldots, i_{k}}=-\frac{K !}{(N-1) !} \log A$

with $A$ a constant.

In combination with $K-1$ reduction relations linking $G_{N}^{(1)}$ to $G_{N}^{(2)}$ to ... to $G_{N}^{(K)}$ and with the normalisation of $G_{N}^{(1)}$, this yields a set of $(K+1)$ equations for the $K$ functions $G_{N}^{(1)}$ to $G_{N}^{(K)}$ and for the constant $A$. 
This set of equations yields an approximate description of the system at thermodynamic equilibrium, but it is completely useless for practical calculations because of the complicated way that the system size $(\mathrm{N}, \mathrm{V})$ is involved. This means that, after choosing the order of the approximation $\mathrm{K}$, the asymptotic limit of large $\mathrm{N}$ and $\mathrm{V}$, with $\rho=\mathrm{N} / \mathrm{V}$ fixed, must be taken in the resulting set of equations.

Before discussing this for the case $K=3$, however, we note that the constant $A$ is related to the free energy in equilibrium. Combining Eq. (13) with Eq. (11) we find that the minimum or equilibrium value of $\mathrm{F}_{\mathrm{N}}^{(\mathrm{K})}$ can be written as

$\min \beta F_{N}^{(K)}=3 N \log \Lambda+N \log \rho-N \log A$

In principle all other thermodynamic functions can be calculated from this free energy. Thus, this approach yields an approximate fluid theory that is completely self-consistent, since all information is obtained from a single approximate free energy functional.

We now choose $\mathrm{K}=3$ in the foregoing, that is we neglect all correlations that involve groups of four or more particles. The set of equations that describes thermodynamic equilibrium in this approximation is found from Eqs. (13) and (14), combined with reduction- and normalisation relations. This set of equations has an asymptotic form that is valid for $\mathrm{N} \rightarrow \infty, \mathrm{V} \rightarrow \infty$ with $\rho=\mathrm{N} / \mathrm{V}$ fixed. The calculation of the asymptotic form involves a considerable amount of algebra and is given in (Schlijper \& Harris, 1991).

For a system without three-particle interactions, i.e. $\Phi_{123} \equiv 0$, the final equations are written in terms of the local density and the pair distribution function as:

$\log g_{12}=-\beta \Phi_{12}+\int d \vec{r}_{3} \rho_{3}\left(g_{13}-1\right)\left(g_{32}-1\right)$

$\log \rho_{1}=-\beta \Phi_{1}+\beta \mu-3 \log \Lambda-1 / 3$

$$
\begin{aligned}
& +\int d \vec{r}_{2} \rho_{2}\left(g_{12}-1\right) \\
& -\frac{1}{2} \int d \vec{r}_{2} \rho_{2} g_{12}\left(\beta \Phi_{12}+\log g_{12}\right) \\
& -\frac{1}{2} \int d \vec{r}_{2} \int d \vec{r}_{3} \rho_{2} \rho_{3}\left(g_{13}-1\right)\left(g_{32}-1\right)
\end{aligned}
$$

$\mu$ in Eq. (15b) is the chemical potential, calculated from Eq. (14).

The twofold integration in the last term can be reduced to a single integration by 
using Eq. (15a). Nonetheless, solution of these non-linear integral equations for $\rho^{(1)}$ and $g^{(2)}$, given $\beta, \mu$ and the potentials $\Phi^{(1)}$ and $\Phi^{(2)}$ is of course a formidable task. In the next section we consider a simplified version of the triplet theory, where we use an additional approximation to decouple the two integral equations; we will present numerical results for this simplified version of the equations.

It may be important to remark that the structure of this CVM-type approximation is such that the one-body functions (like local density) are predicted more accurately than two-body functions (like $\mathrm{g}(\mathrm{r})$ ), which in turn are predicted more accurately than three-body functions. Hence, we expect Eq. (15b) to contain more useful information than Eq. (15a). E.g., the radial distribution function of a bulk hard-sphere fluid is not predicted very well at all from Eq. (15a). To obtain an accurate equation for the pair distribution function in this approach it is probably necessary to keep four terms in the expansion of the free energy.

\section{A SIMPLE THEORY FOR WEAKLY INHOMOGENEOUS FLUIDS}

To introduce a decoupling approximation into the set of equations (15) we consider the bulk fluid with density $\rho_{b}$ and pair distribution function $g_{12}$ that would be in thermodynamic equilibrium (and thus have the same $\beta$ and $\mu$ ) with an inhomogeneous fluid. We write Eq. (15b) for this bulk fluid and subtract the resulting equation for $\log \rho_{h}$ from the equation for $\log \rho_{1}$ as it stands. This eliminates $\mu$ as parameter, and introduces $\rho_{b}$ in its place. Now we make the simplifying approximation that the pair distribution function of the inhomogeneous fluid equals the pair distribution function of the coexisting bulk fluid. This will be a reasonable approximation for weakly inhomogeneous fluids; in terms of the function

$\delta(\vec{r})=\frac{\rho^{(1)}(\vec{r})}{\rho_{b}}-1$

this means we consider the case where $\delta$ is small.

We then have the following equation:

$$
\begin{aligned}
& \log \left(1+\delta_{1}\right)=-\beta \Phi_{1}+\rho_{\mathrm{b}} \int \mathrm{d} \overrightarrow{\mathrm{r}}_{2} \delta_{2}\left(\mathrm{~g}_{12}^{\mathrm{b}}-1\right) \\
& \quad-\frac{1}{2} \rho_{\mathrm{b}} \int \mathrm{d} \overrightarrow{\mathrm{r}}_{2} \delta_{2} \mathrm{~g}_{12}^{\mathrm{b}}\left(\beta \Phi_{12}+\log \mathrm{g}_{12}^{\mathrm{b}}\right) \\
& \quad-\frac{1}{2} \rho_{\mathrm{b}}^{2} \int \mathrm{d} \overrightarrow{\mathrm{r}}_{2} \int \mathrm{d} \overrightarrow{\mathrm{r}}_{3}\left(\delta_{2}+\delta_{3}+\delta_{2} \delta_{3}\right)\left(\mathrm{g}_{13}^{\mathrm{b}}-1\right)\left(\mathrm{g}_{32}^{\mathrm{b}}-1\right)
\end{aligned}
$$


With the abbreviations

$C_{12}^{b}=\int d \vec{r}_{3}\left(g_{13}^{b}-1\right)\left(g_{32}^{b}-1\right), \quad Q=\int d \vec{r}_{2}\left(g_{12}^{b}-1\right)$

and noting that the $g_{i j}^{b}$ are generally functions of $\left\|\vec{r}_{j}-\vec{r}_{j}\right\|$ only, we can rewrite the last term in Eq. (17) to obtain, after neglecting a term that is quadratic in the (supposedly small) quantity $\delta(\vec{r})$, the following equation:

$\log \left(1+\delta_{1}\right)=-\beta \Phi_{1}+\left(1-\rho_{b} \cdot \frac{Q}{2}\right) \rho_{b} \int d \vec{r}_{2} \delta_{2}\left(g_{12}^{b}-1\right)$

$$
-\frac{1}{2} \rho_{b} \int d_{2} \delta_{2}\left\{g_{12}^{b}\left(\beta \Phi_{12}+\log g_{12}^{b}\right)+\rho_{b} C_{12}^{b}\right\}
$$

This equation yields the density profile if the external potential and the pair distribution function of the bulk fluid are given.

Note that in deriving this simple theory we used only Eq. (15b) but not Eq. (15a). It is thus permissible to obtain $g_{12}$ from any source, whether it be the bulk version of Eq. (15a) or an equation of proven value like Percus-Yevick (PY) or hypernetted chain (HNC), or computer simulation data. However, the chemical potential that is consistent with this simple theory is the one implied by Eq. (15a):

$\beta \mu-3 \log \Lambda-1 / 3=\log \rho_{b}-\rho_{b} Q+\frac{1}{2} \rho_{b}^{2} Q^{2}+\frac{1}{2} \rho_{b} \int d \vec{r}_{2} g_{12}^{b}\left(\beta \Phi_{12}+\log g_{12}^{b}\right)$

The term $1 / 3$ is the result of truncating the free energy expansion. It is a shift of numerical values that is without physical consequences. 


\section{RESULTS AND DISCUSSION}

We have applied Eq. (19) to calculate the density profiles of hard sphere and Lennard-Jones fluids confined to the space between two plane parallel hard walls. We obtained the bulk pair distribution function from the PY equation. We also tried to obtain $\mathbf{g}_{12}$ from our triplet theory (i.e. Eq. (15a) for a bulk fluid), but ran into numerical problems for the Lennard-Jones fluid. Some results for hard spheres have been reported by Schlijper and Harris (1991).

Figure 1 shows the density profile of the hard-sphere fluid at bulk density $\rho_{b} \sigma^{3}=0.5$ in a slit where the distance $L$ between the walls is $4.5 \sigma$, with $\sigma$ the molecular diameter. The distance between the hard walls is defined as the width of the region available to the centers of the molecules, i.e. the external potential is $\Phi_{\text {ext }}(z)=0$ if $|z|<L / 2$ and $=+\infty$ if $|z| \geq L / 2$. The computer simulation results are taken from the work of Antonchenko et. al. (1984).

Figure 2 shows the density profile for a Lennard-Jones fluid at an average density $\rho_{\mathrm{av}} \sigma^{3}=0.65$ and $\varepsilon / \mathrm{kT}=0.4$, with $\sigma$ and $\varepsilon$ the Lennard-Jones parameters, in a wide slit $(L=8 \sigma)$. The potential is truncated at $R_{c}=4 \sigma$ and shifted to have it go to zero continuously. The computer simulation results are our own. Figure 3 shows the same information for $\rho_{\mathrm{av}} \sigma^{3}=0.5, \varepsilon / \mathrm{kT}=0.75, \mathrm{~L}=12 \sigma$ and $\mathrm{R}_{\mathrm{c}}=2.5 \sigma$. The qualitative shape of the profile is typical of Lennard-Jones fluids at a hard wall. Density-functional theories that treat molecular attraction in a mean-field approximation apparently have difficulty describing this behaviour (Tang et al., 1991). The simple Eq. (19) is able to describe qualitatively quite different profiles correctly in spite of the crude approximation $g_{12}=g_{12}$ on which it is based.

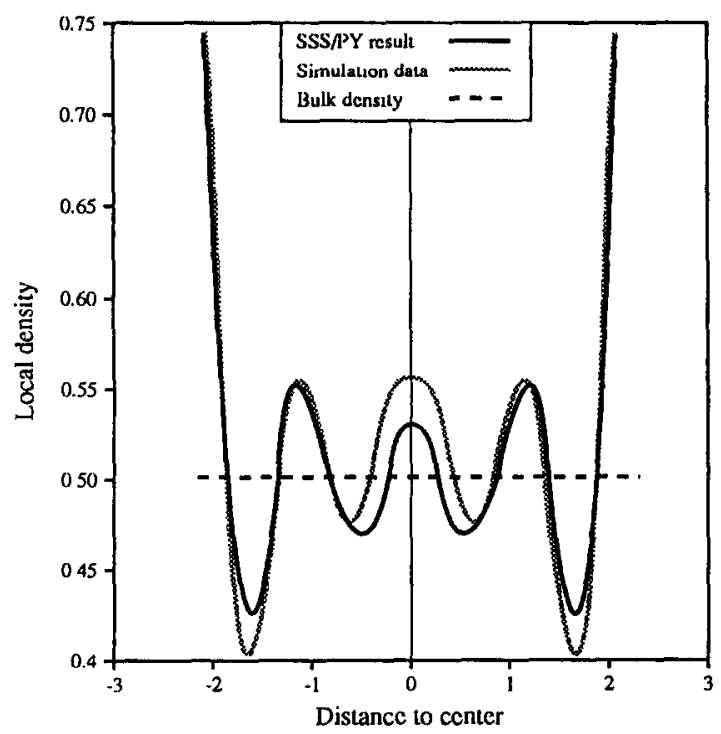

Fig. 1. Density profile of hard sphere fluid in hard slit, slit width $4.5 \sigma$, bulk density $0.5 \sigma^{-3}$ 


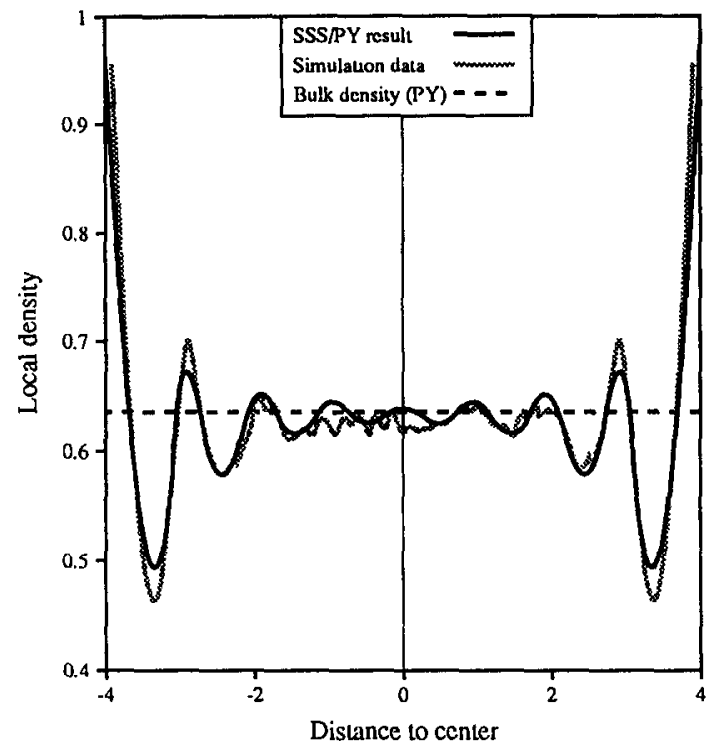

Fig. 2. Density profile of Lennard-Jones fluid in hard slit, slit width $8 \sigma$, average density $0.65 \sigma^{-3}, \varepsilon / \mathrm{kT}=0.4$

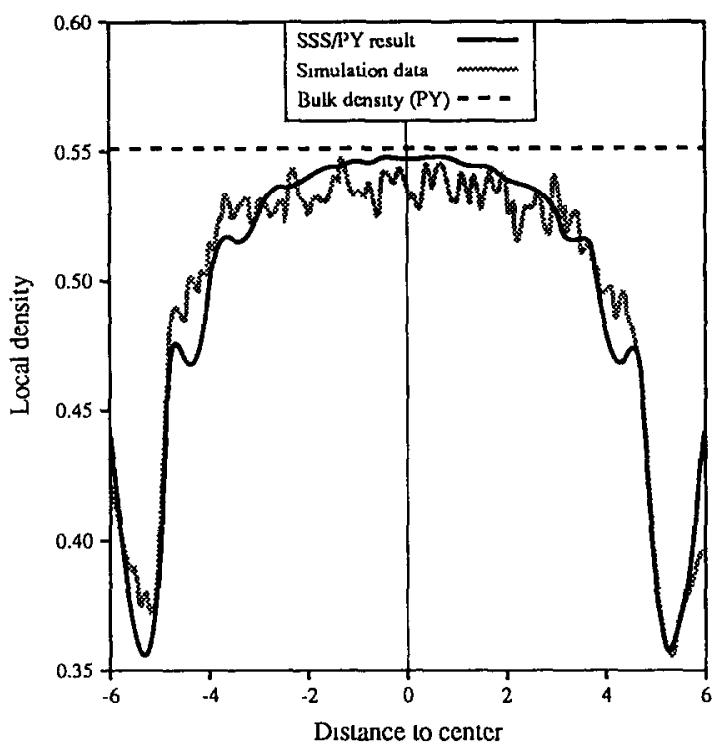

Fig. 3. Density profile of Lennard-Jones fluid in hard slit, slit width $12 \sigma$, average density $0.5 \sigma^{-3}, \varepsilon / \mathrm{kT}=0.75$ 
Since we neglected the term quadratic in $\delta(\vec{r})$ in going from Eq. (17) to Eq. (19), Eq. (19) cannot accommodate two-phase coexistence. Eq. (17) has the capability in principle, even though it rests on the crude assumption $\mathrm{g}_{12}=\mathrm{g}_{12}$, since it can support three homogeneous solutions. Whether it actually can be employed to describe wetting and drying will be investigated in the future.

Other future efforts will be aimed at deriving the full quartet theory. While we fully expect the quartet theory to be unwieldy, we have good hope that it will prove amenable to simplifications like the one employed here in the triplet theory.

In conclusion, we believe that the adaptation of the cluster-variation method to inhomogeneous fluids provides an interesting alternative to the density functional approach. The results shown here encourage further investigation.

\section{REFERENCES}

Antonchenko, V.Y., Ilyin, V.V., Malcovsky, N.N., Pavlov, A.N. and Sokhan, V.P., 1984. On the nature of disjoining pressure oscillations in fluid films. $\mathrm{Mol}$. Phys., 52: 345- 355.

An, G.Z., 1988. A note on the Cluster Variation Method, J. Stat. Phys., 52: 727734.

Kikuchi, R., 1951. A theory of cooperative phenomena. Phys. Rev., 81: $988-1003$.

Rota, G.C., 1964. On the foundations of combinatorial theory. I. Theory of Möbius functions. Z. Wahrsch, 2: 340-368.

Schlijper, A.G., 1983. Convergence of the cluster-variation method in the thermodynamic limit. Phys. Rev., B27: 6841-6848.

Schlijper, A.G. and Harris, C.K., 1991. Distribution function theory for inhomogeneous fluids. J. Chem. Phys., 95: 7603-7611.

Schlijper, A.G. and Kikuchi, R., 1990. A variational approach to distribution function theory. J. Stat. Phys. 61: 143-160.

Tang, Z., Scriven, L.E. and Davis, H.T., 1991. Density-functional perturbation theory of inhomogeneous simple fluids. J. Chem. Phys., 95: 2659-2668. 